\title{
“Aprender juntos para trabalhar juntos": competências colaborativas desenvolvidas por integrantes de um grupo tutorial do pet-saúde interprofissionalidade
}

\author{
"Learning together to work together": collaborative competences developed by members of a pet- \\ health interprofissionality tutorial group
}

“Aprendiendo juntos a trabajar juntos": competencias colaborativas desarrolladas por miembros de un grupo tutorial de interprofisionalidad pet-salud

\section{Resumo}

Estudo com o objetivo de descrever as competências desenvolvidas pelos integrantes de um grupo tutorial do PETSaúde Interprofissionalidade, a partir das ações realizadas no primeiro ano de implementação do Projeto. Trata-se de um estudo descritivo, do tipo relato de experiência, desenvolvido pelo grupo tutorial do pet interprofissional do município de Sobral, no qual está vinculado a Universidade Estadual Vale do Acaraú, Universidade Federal do Ceará e a Escola de Saúde Pública Visconde de Sabóia, através da Secretaria de Saúde de Sobral. A experiência retrata as ações desenvolvidas no período desde Abril de 2019 até Agosto de 2020 por 14 integrantes, sendo dois tutores, quatro preceptores e oito monitores dos cursos de enfermagem, educação física, medicina, odontologia e psicologia, por meio de rodas de conversa no grupo tutorial, onde foi feito uma revisão ao Projeto Pet Saúde Interprofissionalidade Sobral e o relatório semestral, elencando as competências identificadas e classificando-as em específicas, comuns e colaborativas. A experiência da integração das diferentes formações tornou-se possível identificar as ações desenvolvidas de forma perceptível, onde foi manifestada pelos monitores, preceptores e tutores nos relatos das ações e enquadrá-las de acordo com as competências: específicas, comuns e colaborativas, desenvolvidas com os discentes e profissionais de saúde. A participação no PET- Saúde- Interprofissional, as diretrizes das competências foram potencializadas em meio às ações realizadas pelos participantes entre si e juntos ao sistema de saúde. Portanto, é de relevância na prática interprofissional, que os ideais das competências sejam implementados integralmente na perspectiva da interprofissionalidade.

Palavras-chave: Educação interprofissional; Competências colaborativas; Formação e trabalho em saúde; Relações interprofissionais; Integralidade em saúde. 


\begin{abstract}
Objective: To describe the skills developed by the members of a group tutorial of PET-Health Interprofessionality, based on the actions carried out in the first year of implementation of the Project. Method: This is a descriptive study of the experience report type, developed by PET tutorial group of Sobral city, in which is linked to the Vale do Acaraú State University, Federal University of Ceará and the School of Public Health Visconde de Sabóia, through the Sobral Health Department. The Experience portrays the actions developed by 14 members, Wich is formed by two tutors, four preceptors and eight monitors from the courses of Nursing, Physical Education, Medicine, Dentistry and Psychology, through conversation groups in the tutorial group, where a revision was made to the Pet Health Interprofessionality Project Sobral and the semiannual report, listing the competencies identified and classifying them into specific, common and collaborative, systematizing the information in a chart. Results: The experience of integrating different formations it became possible to identify the actions developed in a perceptible way, where it was manifested by the monitors, preceptors and tutors in the reports of actions and classify them according to the competencies: specific, common and collaborative, developed with students and health professionals. Conclusion: With the current participation of PET- Health - Interprofessional, the guidelines of the competencies were potentiated in the midst of the actions carried out by participants among themselves and together with the health system. Therefore, it is of relevance in interprofessional practice, that the ideals of competences are implemented fully from the perspective of interprofessionality, facilitating and strengthening the collaborative work.
\end{abstract}

Keywords: Interprofessional relations; Professional competence; Integrality in health.

\begin{abstract}
Resumen
Objetivo: Describir las habilidades desarrolladas por los miembros de um grupo tutorial de Interprofesionalidad PETSalud, basado en las acciones realizadas en el primer año de implementación del Proyecto. Método: este es um estudio descriptivo, del tipo informe de experiencia, desarrollado por el grupo de tutoría de PET interprofesional del municipio de Sobral, en el que está vinculada la Universidad Estatal Vale do Acaraú, Universidad Federal de Ceará y Escuela de Salud Pública Visconde de Sabóia, através del Departamento de Salud de Sobral. La experiencia retrata las acciones desarrolladas por 14 integrantes, siendo dos tutores, cuatro preceptores y ocho monitores de los cursos de enfermería, educación física, medicina, odontología y psicología, através de grupos de conversación en el grupo tutorial, donde se hizo uma revisión al Proyecto de PET Interprofesionalidad em Salud de Sobral y el informe semestral, enumerando las competencias identificadas y clasificándolas en específicas, comunes y colaborativas, sistematizando la información em uma pizarra. Resultados: Con la experiencia de integrar diferentes formaciones se hizo posible identificar las acciones desarrolladas en una forma perceptible, donde fue manifestado por los monitores, preceptores y tutores en los informes acciones y enmarcarlas de acuerdo com las competencias: específicas, comunes y colaborativas, desarrollado com estudiantes y profesionales de la salud. Conclusión: Con la participación actual de PET- Salud- Interprofessional, los lineamientos de las competencias se potenciaron en medio de las acciones realizadas por participantes entre ellos y junto con el sistema de salud. Por tanto, es de relevancia en la práctica interprofesional, que se implementen los ideales de competencias plenamente desde la perspectiva de la interprofesionalidad, facilitando y fortaleciendo el trabajo colaborativo.
\end{abstract}

Palabras clave: Relaciones interprofesionales; Competencia professional; Integralidad en salud.

\title{
1. Introdução
}

A partir do movimento sanitário brasileiro na década de 80, viu-se a necessidade de reformar o setor saúde. Com isso resultou na criação do Sistema Único de Saúde (SUS), e no estabelecimento de novas diretrizes e princípios a partir da Lei 8080/90, principalmente, voltado à configuração de um novo modelo de saúde (Paiva \& Teixeira, 2014). Nesse cenário de indução de políticas públicas que apoiasse a operacionalização do SUS,

Concomitantemente a esse cenário, houve a necessidade da criação de estratégias para reorientar a formação e o trabalho em saúde. Em prosseguimento a esse processo, foi regulamentado pela Portaria Interministerial $n^{\circ} 421$, de 3 de março de 2010, o Programa de Educação pelo Trabalho para a Saúde (PET - Saúde), que visa estabelecer a educação pelo trabalho, tendo perspectivas a inserção nos serviços, de acordo com as necessidades do SUS, caracterizando-o como fonte de conhecimento e pesquisa nas instituições de ensino, dirigidos aos estudantes dos cursos de graduação, pós-graduação e aos profissionais da saúde (Batista et al., 2015).

Nessa perspectiva, a formação em saúde no Brasil baseada na lógica uniprofissional se encontra em desuso e vem se destacando a Educação Interprofissional em Saúde (EIP), contribuindo para a qualificação dos profissionais de saúde e a formação de estudantes das graduações. A EIP surge como uma estratégia de qualificar a formação e o trabalho em saúde, a 
partir da orientação para um trabalho colaborativo em equipe interprofissional, além de ter um cuidado mais ampliado e de forma integral. Na EIP, as profissões aprendem conjuntamente sobre o trabalho coletivo e possuem como pilares o aprender "com", "sobre" e "entre si", atuando integralmente e compartilhando saberes com diversas categorias profissionais (Costa et al., 2018).

Nesse contexto que envolve a interprofissionalidade e a prática colaborativa, se insere a edição 2018/2019 do Programa de Educação pelo Trabalho para a Saúde - PET Saúde / Interprofissionalidade (Ministério da Saúde, 2018), que visa estimular mudanças curriculares para os cursos de graduação da área da saúde alinhados aos princípios da interprofissionalidade, intersetorialidade e interdisciplinaridade; além de qualificar os processos de integração ensino-serviçocomunidade de modo a promover mudanças nas formações dos profissionais e desenvolvimento do trabalho colaborativo (Almeida et al., 2019).

No sistema de saúde vigente, alinhar as diferentes formações ainda é um desafio, pois conforme Lima et al. (2018), “as atividades educacionais voltadas para profissionais da saúde ainda são predominantemente organizadas segundo essa lógica disciplinar ou de acordo com a perspectiva de especialistas ou categorias profissionais" (p. 1551). Desse modo, a individualidade da profissão, caracterizada por seus conhecimentos e percepção distinta do outro, faz com que haja necessidade da complementaridade dos saberes para o desenvolvimento do trabalho interprofissional, tendo em vista uma melhor garantia do atendimento das necessidades de cuidado da população assistida.

Essa realidade já vem sendo discutida no cenário brasileiro, em virtude de que, para o sistema de saúde contemplar a integralidade, universalidade e as diferentes necessidades da população, faz-se necessário pensar em um modelo de formação acadêmica que seja fundamentada na concepção ampliada de saúde. Isto posto, enquanto o modelo vigente no sistema de saúde corroborar para uma organização de trabalho em que o conhecimento especializado prevalece, questiona-se a efetividade da lógica: problema-solução, presente nos serviços de assistência à saúde (Ferla \& Toassi, 2017).

Nos currículos da saúde, a proposta de formação uniprofissional, predominante nas diretrizes curriculares, direciona para uma formação de competências específicas, centrada na particularidade de determinada área de conhecimento, consequentemente haverá prejuízos na oferta dos serviços para a comunidade, pois serão práticas de saúde fragmentadas (Costa, 2017).

Haja vista a necessidade da integralidade do cuidado e as dificuldades encontradas na articulação de ações entre os profissionais atuantes do sistema de saúde, pontua-se a urgência de mudanças no processo de produção dos serviços, visando o fortalecimento do trabalho colaborativo em equipes multiprofissionais, nos diferentes cenários, para o aprimoramento dos serviços de atenção à saúde (Silva et al., 2015).

A educação interprofissional se propõe a formar profissionais de saúde mais aptos ao efetivo trabalho em equipe. Profissionais colaborativos asseguram práticas em saúde integrais por meio do trabalho colaborativo, com maior capacidade de respostas aos problemas e às necessidades de saúde (Reeves, 2016). Os profissionais têm como missão colocar em prática as competências que garante a mudança no nosso sistema de saúde, seguindo o modelo de atenção à saúde: a colaboração.

Desse modo, as competências colaborativas têm em sua função, primordialmente, a contribuição e o favorecimento da colaboração, uma vez que ela só pode ser colocada em ação mediante a dois ou mais profissionais de distintas categorias na realização dos procedimentos. À vista disso, dentre as atividades que contemplam as competências colaborativas, destacam-se: planejamento conjunto de planos terapêuticos, capacidade de argumentação e negociação e o respeito às especificidades de cada profissão. Em virtude do que foi mencionado, o modelo supracitado tem em seu papel, a fundamentação da clareza de papéis, liderança colaborativa, dinâmica de equipe, atenção centrada ao paciente e uma melhor resolução de conflitos interprofissionais (Silva et al., 2015). 
Visto que a formação por competências tem por finalidade qualificar os profissionais na resolução dos problemas, por meio da adesão das competências para o aprender a aprender, acontece uma absorção dos conhecimentos, atitudes e habilidades. Ressalta-se a importância de estudá-las, uma vez que são direcionadas na contribuição da mudança no modelo da atenção e da formação dos profissionais de saúde na perspectiva da integralidade do trabalho em equipe, da comunicação e da prática colaborativa.

Desta forma, a realização das ações no primeiro ano do grupo tutorial foram direcionadas para a necessidade de aproximação dos estudantes e dos trabalhadores da saúde no cenário das práticas com a educação interprofissional e com competências colaborativas e comuns para atuarem no sistema único de saúde, pois, compreende-se que o conviver interativo, são potenciais para o desenvolvimento das competências.

Destarte, este estudo foi desenvolvido com o objetivo de descrever as competências desenvolvidas pelos integrantes de um grupo tutorial do PET-Saúde Interprofissionalidade, a partir das ações realizadas no primeiro ano de implementação do Projeto.

\section{Metodologia}

Trata-se de um estudo descritivo, do tipo relato de experiência, com abordagem qualitativa, sistematizado pelo grupo tutorial do pet interprofissional do município de Sobral, no qual está vinculado a Universidade Estadual Vale do Acaraú, Universidade Federal do Ceará e a Escola de Saúde Pública Visconde de Sabóia, através da Secretaria de Saúde de Sobral. Gil (2002), afirma que a pesquisa classificada como descritiva, tem por finalidade descrever os fenômenos ou particularidades de uma determinada realidade. Assim como, segundo Pereira et al (2018), no método qualitativo, as interpretações que o pesquisador realiza do fenômeno estudado são relevantes para a pesquisa.

Um relato de experiência consiste em um tipo de estudo cujo objeto é a própria experiência descrita, a qual constitui uma fonte de sentidos e possibilidades passíveis de interpretações e análises dos sujeitos envolvidos (Daltro \& Faria, 2019).

O estudo retrata as ações desenvolvidas por 14 integrantes, sendo dois tutores, quatro preceptores e oito monitores dos cursos de enfermagem, educação física, odontologia e psicologia, por meio de rodas de conversa no grupo tutorial, onde elencou-se as competências identificadas, classificando-as em específicas, comuns e colaborativas.

As ações foram sistematizadas em um quadro durante o período de julho a setembro de 2020, considerando as competências definidas pelo grupo de trabalho, e, posteriormente, foram discutidas à luz da literatura em educação interprofissional e prática colaborativa, no sentido de estimular o desenvolvimento de competências colaborativas tanto no espaço da formação quanto do trabalho em saúde e assim, contribuir para a melhoria da qualidade dos serviços no SUS. O estudo incorporou os princípios da Resolução 466/2012 do Conselho Nacional de Saúde que trata de pesquisa envolvendo seres humanos.

\section{Resultados}

As ações realizadas pelos participantes de um determinado grupo tutorial do PET-Saúde Interprofissionalidade Sobral, foram sistematizadas e analisadas a partir da literatura acerca das competências colaborativas para o trabalho em equipe atrelando a uma aprendizagem significativa. $O$ Quadro 1 mostra as ações desenvolvidas no primeiro ano implementação do Projeto 2019/2020 e as competências desenvolvidas pelos integrantes do grupo tutorial. 
Quadro 1 - Ações realizadas pelos integrantes de um grupo tutorial do PET-Saúde Interprofissionalidade e competências desenvolvidas durante o primeiro ano de implementação do Projeto. Sobral, Ceará, 2019-2020.

\begin{tabular}{|c|c|c|c|}
\hline \multirow{2}{*}{ AÇÕES } & \multicolumn{3}{|c|}{ COMPETÊNCIAS } \\
\hline & Comuns & Específicas & Colaborativas \\
\hline $\begin{array}{l}\text { Curso sobre Educação Interprofissional } \\
\text { em Saúde para alinhamento conceitual }\end{array}$ & $\begin{array}{c}\text { Tomada de decisões; } \\
\text { Comunicação; Educação } \\
\text { permanente }\end{array}$ & $\begin{array}{l}\text { Não se } \\
\text { aplica }\end{array}$ & $\begin{array}{l}\text { Funcionamento da equipe; Liderança } \\
\text { colaborativa; Comunicação } \\
\text { Interprofissional; Resolução de conflitos } \\
\text { interprofissionais }\end{array}$ \\
\hline $\begin{array}{l}\text { Rodas de conversa com estudantes e } \\
\text { docentes dos NDE dos cursos envolvidos } \\
\text { no PET para refletir sobre a } \\
\text { implementação da EIP e PC no currículo }\end{array}$ & $\begin{array}{c}\text { Atenção à Saúde; } \\
\text { Tomada de decisões; } \\
\text { Comunicação; } \\
\text { Administração e } \\
\text { gerenciamento; Educação } \\
\text { permanente }\end{array}$ & $\begin{array}{l}\text { Não se } \\
\text { aplica }\end{array}$ & $\begin{array}{c}\text { Clareza dos papéis; Funcionamento da } \\
\text { equipe; Liderança colaborativa; Atenção } \\
\text { centrada no paciente/família/comunidade; } \\
\text { Comunicação Interprofissional; Resolução } \\
\text { de conflitos interprofissionais }\end{array}$ \\
\hline $\begin{array}{l}\text { Intervenção junto à equipe de saúde em } \\
\text { Centros de Saúde da Família sobre o } \\
\text { trabalho colaborativo em equipe }\end{array}$ & $\begin{array}{l}\text { Comunicação; Educação } \\
\text { permanente. }\end{array}$ & $\begin{array}{l}\text { Não se } \\
\text { aplica }\end{array}$ & $\begin{array}{l}\text { Comunicação Interprofissional; } \\
\text { Resolução de conflitos interprofissionais }\end{array}$ \\
\hline $\begin{array}{l}\text { Participação no OUTUBRO UVA, } \\
\text { motivando participantes do evento a } \\
\text { refletirem e escreverem em Painel } \\
\text { Interativo expressando o entendimento } \\
\text { sobre Interprofissionalidade }\end{array}$ & $\begin{array}{c}\text { Atenção à Saúde; } \\
\text { Comunicação; Educação } \\
\text { permanente }\end{array}$ & $\begin{array}{l}\text { Não se } \\
\text { aplica }\end{array}$ & $\begin{array}{l}\text { Clareza dos papéis; } \\
\text { Funcionamento da equipe; Comunicação } \\
\text { Interprofissional }\end{array}$ \\
\hline $\begin{array}{l}\text { Construção do } \quad \text { Varal da } \\
\text { Interprofissionalidade } \\
\text { estudantes dos cursos de Enformagem e } \\
\text { Educação Física - Estratégia educacional } \\
\text { desenvolvida em sala de aula sobre } \\
\text { interprofissionalidade recorrendo-se a } \\
\text { metodologias ativas }\end{array}$ & $\begin{array}{l}\text { Comunicação; Educação } \\
\text { permanente; }\end{array}$ & $\begin{array}{l}\text { Não se } \\
\text { aplica }\end{array}$ & $\begin{array}{l}\text { Comunicação Interprofissional; } \\
\text { Funcionamento da equipe; } \\
\text { Clareza dos papéis }\end{array}$ \\
\hline $\begin{array}{l}\text { Edição de um vídeo com a finalidade de } \\
\text { divulgar o Projeto Pet-Saúde } \\
\text { Interprofissionalidade e as inúmeras } \\
\text { ações desenvolvidas por ele nos nossos } \\
\text { espaços de atuação }\end{array}$ & $\begin{array}{c}\text { Comunicação; Educação } \\
\text { permanente }\end{array}$ & $\begin{array}{l}\text { Não se } \\
\text { aplica }\end{array}$ & $\begin{array}{c}\text { Funcionamento da equipe; } \\
\text { Comunicação Interprofissional }\end{array}$ \\
\hline $\begin{array}{l}\text { Produção de um Infográfico sobre EIP e } \\
\text { PC com o objetivo de apresentar a } \\
\text { diferenciação entre os conceitos } \\
\text { interprofissionalidade, } \\
\text { multiprofissionalidade, trabalho em } \\
\text { equipe, interdisciplinaridade, entre outros, } \\
\text { com vistas a promover um alinhamento } \\
\text { conceitual }\end{array}$ & $\begin{array}{l}\text { Educação permanente; } \\
\text { Tomada de decisões; } \\
\text { Comunicação }\end{array}$ & $\begin{array}{l}\text { Não se } \\
\text { aplica }\end{array}$ & $\begin{array}{l}\text { Funcionamento da equipe; } \\
\text { Liderança colaborativa; } \\
\text { Comunicação Interprofissional }\end{array}$ \\
\hline $\begin{array}{l}\text { Processo de sistematização das } \\
\text { experiências vivenciadas pelo grupo e } \\
\text { submetidos em periódicos }\end{array}$ & $\begin{array}{l}\text { Educação permanente; } \\
\text { Tomada de decisões; } \\
\text { Comunicação }\end{array}$ & $\begin{array}{l}\text { Não se } \\
\text { aplica }\end{array}$ & $\begin{array}{l}\text { Funcionamento da equipe; } \\
\text { Liderança colaborativa; } \\
\text { Comunicação Interprofissional }\end{array}$ \\
\hline $\begin{array}{l}\text { Elaboração dos capítulos para o Ebook } \\
\text { sobre Educação Interprofissional em } \\
\text { Saúde e Prática Colaborativa }\end{array}$ & $\begin{array}{l}\text { Educação permanente; } \\
\text { Comunicação }\end{array}$ & $\begin{array}{l}\text { Não se } \\
\text { aplica }\end{array}$ & $\begin{array}{l}\text { Clareza dos papéis; Funcionamento da } \\
\text { equipe; Liderança colaborativa; } \\
\text { Comunicação Interprofissional; } \\
\text { Resolução de conflitos interprofissionais }\end{array}$ \\
\hline
\end{tabular}


Durante o primeiro ano de desenvolvimento das atividades do programa tivemos a oportunidade de realizarmos algumas atividades para divulgar o trabalho do PET e trazer à tona na comunidade acadêmica a discussão sobre os elementos essenciais da Interprofissionalidade e da Prática Colaborativa. Tivemos a oportunidade fazer essas discussões junto a estudantes, professores e profissionais do serviço, participantes e não participantes do PET.

Refletir sobre as experiências exitosas que marcaram a participação dos profissionais e estudantes da saúde durante o primeiro ano de execução do programa PET Saúde Interprofissionalidade, proporcionou resgatar os conceitos de educação interprofissional em saúde e suas implicações para a efetivação do trabalho em equipe, consequentemente, o desenvolvimento de competências necessárias para o fortalecimento do trabalho colaborativo. Visto que o programa visa habilitar ações de integração ensino-serviço-comunidade, englobando os atores do SUS e da comunidade acadêmica, além de promover ações que estimulem o desenvolvimento das competências colaborativas por meio da interprofissionalidade, interdisciplinaridade e intersetorialidade (Ministério da Saúde, 2018).

O grupo Canadian Interprofessional Health Collaborative (CIHC), estabeleceu seis domínios de competências essenciais para a prática interprofissional colaborativa, sendo eles: cuidado centrado no paciente, cliente, família e comunidade; clarificação de papéis profissionais; comunicação interprofissional; dinâmica de funcionamento da equipe; resolução de conflitos interprofissionais e liderança colaborativa. Tais domínios fornecem uma abordagem integrativa para descrever as competências necessárias para um trabalho interprofissional e colaborativo, destacando os conhecimentos, habilidades, atitudes e valores que moldam os julgamentos essenciais para a colaboração interprofissional (Canadian Interprofessional Health Collaborative [CIHC], 2010).

Conforme observado no quadro 1, as competências específicas abrangem o desenvolvimento das ações em que o conhecimento específico de cada profissão deve ser compartilhado entre os demais profissionais da saúde, para que atenda as necessidades dos usuários do sistema de saúde, e o processo de trabalho em saúde seja caracterizado pela colaboração interprofissional. Entretanto, quando os profissionais são ensinados numa formação com ênfase nas competências específicas, legitimam práticas em saúde fragmentadas, expondo o usuário a atendimentos duplicados, levando ao retrabalho da equipe multiprofissional, logo, gera aumento nos riscos de erros e gastos em saúde (Freire et al., 2019). Portanto, há uma necessidade de formar profissionais de saúde com competências para execução do trabalho em equipe, e estes estejam aptos à colaboração.

Com relação às competências colaborativas, é necessário o compartilhamento de conhecimentos entre as diversas áreas da saúde, no qual estimulem o aprender com outros profissionais e a também ensinar, objetivando ações centradas nas necessidades de cuidado do indivíduo.

Quanto às competências comuns que envolvem o planejamento e a integração ensino-serviço-comunidade; no programa PET-Saúde Interprofissionalidade pode ser identificada nos conhecimentos apreendidos nos estudos realizados durante as reuniões nos grupos tutoriais e outras formações estruturadas por cada eixo do programa, em que ocorre articulação dos saberes, habilidades e atitudes com outros atores que não integram diretamente no PET.

A competência colaborativa que merece destaque é a clarificação de papéis dos membros da equipe, que apareceu diversas vezes no decorrer da atividade, demonstrando uma visão muito limitada e até mesmo estereotipada das demais áreas profissionais. Com isso, vale ressaltar a importância de promover ações compartilhadas permitindo desenvolver uma visão clara da importância de cada categoria profissional na atenção à saúde, e também na identidade de pertencimento a uma equipe para manter a dinâmica de um trabalho colaborativo (Silva et al., 2015).

Diante disso, são apresentadas pelo menos seis competências em comum nos cursos da área da saúde, dentre elas destacam-se: 
"atenção à saúde": ações de prevenção, promoção, proteção e reabilitação da saúde; "tomada de decisões": uso apropriado dos recursos disponíveis; "comunicação": interação com os profissionais da saúde e com o público, bem como a confidencialidade; "liderança": trabalho em equipe multidisciplinar; "administração e gerenciamento": do SUS; "educação permanente": capacidade de aprender continuamente e "aprender a aprender". (Silveira et al., 2020, pág. 4)

A competência colaborativa que merece destaque é a clarificação de papéis dos membros da equipe, que apareceu diversas vezes no decorrer da atividade, demonstrando uma visão muito limitada e até mesmo estereotipada das demais áreas profissionais. Com isso, vale ressaltar a importância de promover ações compartilhadas permitindo desenvolver uma visão clara da importância de cada categoria profissional na atenção à saúde, e também na identidade de pertencimento a uma equipe para manter a dinâmica de um trabalho colaborativo (Silva et al., 2015).

Os autores destacam ainda que a liderança colaborativa, outra competência colaborativa desenvolvida pela EIP, mostrou-se presente, principalmente relacionado no desenvolvimento de habilidades para motivar um grupo de pessoas a atuar na busca de um objetivo comum, e participarem dos processos. O trabalho colaborativo tem como premissa uma atuação de forma interativa, compartilhando objetivos, reconhecendo o papel e a importância do outro na complementaridade dos processos de saúde.

Barr (1998), em seus estudos sobre competências colaborativas sinaliza que estas devem ser formuladas considerando os diferentes aspectos da prática e da gestão. É relevante afirmar que as competências para colaboração entre profissionais podem ser definidas como a capacidade de descrever e executar os próprios papéis e responsabilidades para e com outras profissões; reconhecendo suas restrições e percebendo as necessidades de um trabalho mais amplo, com vistas a revisar serviços, efetivar mudanças, melhorar padrões, resolver problemas e conflitos na prestação de cuidados e tratamentos. Outro aspecto essencial, destacado por Barr é o trabalho com outras profissões para avaliar, planejar, prestar e revisar o atendimento a pacientes individuais, e dar suporte a cuidadores.

A literatura aponta para a necessidade de uma reorientação na dinâmica da produção dos serviços de saúde, visando o fortalecimento e desenvolvimento do trabalho colaborativo nos diversos cenários, melhorando assim a qualidade da atenção à saúde das populações. Para isso, o desenvolvimento de um trabalho interprofissional é de suma importância visto que busca horizontalizar as relações entre os profissionais, reconhecendo o papel e a importância do outro no processo de trabalho em saúde. Seguindo essa premissa, o desenvolvimento das competências colaborativas quando executadas, melhoram as relações entre as diferentes categorias profissionais na dinâmica do trabalho em saúde e colaboram para a garantia de uma ação interprofissional dos trabalhadores (Costa, 2017).

\section{Considerações Finais}

A necessidade de mudanças no modelo de saúde baseado na lógica uniprofissional gerou o estabelecimento de novas estratégias, que tensionam reconfigurações na formação e no trabalho em saúde. O PET-Saúde Interprofissionalidade se insere nesse contexto oportunizando aos envolvidos, vivências interdisciplinares e interprofissionais por meio do compartilhamento de saberes das distintas profissões que compõem o grupo. Mesmo diante dos obstáculos, a EIP tem ganhado destaque no direcionamento do trabalho em equipe colaborativo e conquistado pautas nos espaços coletivos das instituições formadoras e dos serviços de saúde, tanto no âmbito da gestão quanto da atenção à saúde.

Nesse contexto, merecem destaque as discussões sobre as competências colaborativas desenvolvidas com as ações realizadas durante o primeiro ano de implementação do Projeto, potencializando as possibilidades do trabalho em equipe nos espaços da formação e do trabalho em saúde. 
À vista disso, foram exitosas as ações fundamentadas na metodologia das competências colaborativas, entretanto, nota-se que na literatura ainda há escassez de trabalhos com essa temática. Portanto, com base nos resultados colhidos na pesquisa e o aporte literário disponível, recomenda-se como possíveis temas para pesquisas futuras: a percepção dos estudantes das graduações no campo da saúde sobre o trabalho interprofissional, as vivências dos profissionais de saúde inseridos numa equipe multiprofissional e as dificuldades do trabalho em equipe, e a potencialização do trabalho interdisciplinar dentro das universidades com acadêmicos dos cursos da área da saúde.

\section{Referências}

Almeida, R. G. dos S., Teston, E. F. \& Medeiros, A. de A. (2019). A interface entre o PET-Saúde/Interprofissionalidade e a Política Nacional de Educação Permanente em Saúde. Saúde em Debate, 43(spe1), 97-105. https://dx.doi.org/10.1590/0103-11042019s108

Agreli, H. F., Peduzzi, M. \& Silva, M. C. (2016). Atenção centrada no paciente na prática interprofissional colaborativa. Interface (Botucatu), 20(59), 905-16. $10.1590 / 1807-57622015.0511$

Batista, S. H. S. da S, Jansen, B. Assis, E. Q. de, Senna, M. I. B. \& Cury, G. C. (2015). Formação em Saúde: reflexões a partir dos Programas Pró-Saúde e PET-Saúde. Interface (Botucatu), vol. 19 (1), 743-752. https://doi.org/10.1590/1807-57622014.0996

Barr, H. (1998). Competente para colaborar: Rumo a um modelo baseado em competências para educação interprofissional, Journal of Interprofessional Care, 12: $2,181-187,10.3109 / 13561829809014104$

Brêtas, J. R. da S. \& Pereira, S. R. (2007). Projeto de extensão universitária: um espaço para a formação profissional e promoção da saúde. Trabalho, Educação e Saúde, 5(2), 367- 380. https://doi.org/10.1590/S1981-77462007000200008.

Canadian Interprofessional Health Collaborative. (2010). College of health disciplines. University of British Columbia. Vancouver. ISBN: 978-1-926819-07-5.

Capozzolo, A. A., Casetto, S. J., Imbrizi, J. M., de Oliveira, H. A., Tykanori, K. R. \& Ferreira, Q. M. de F. (2014). Narrativas Na Formação Comum De Profissionais De Saúde. Trabalho, Educação e Saúde, 12 (2), 443-456. https://www.redalyc.org/articulo.oa?id=4067/406756990013

Costa, M. V. (2017). A potência da educação interprofissional para o desenvolvimento de competências colaborativas no trabalho em saúde. In R. F. C. Toassi (org.), Interprofissionalidade e formação na saúde: onde estamos? (pp. 14-27). Porto Alegre: Rede Unida: Série Vivência em Educação na Saúde.

Costa, M. V., Freire, J. R., F., Brandão, C. \& Silva, J. A. M. (2018). A Educação e o trabalho interprofissional alinhados ao compromisso histórico de fortalecimento e consolidação do Sistema Único de Saúde (SUS). Interface (Botucatu), 22, 1507-1510. https://doi.org/10.1590/1807-57622018.0636

Daltro, M. R. \& Faria, A. A. de. (2019). Relato de experiência: Uma narrativa científica na pós-modernidade. Estud. Pesqui. Psicologia. Rio de Janeiro, vol. 19(1), pp. 223-237. https://doi.org/10.12957/epp.2019.43015

Ferla, A. A. \& Toassi, R. F. C. (2017). Formação interprofissional em saúde: um caminho a experimentar e pesquisar. In R. F. C. Toassi (org.), Interprofissionalidade e formação na saúde: onde estamos? (pp. 7-13). Porto Alegre: Rede Unida: Série Vivência em Educação na Saúde.

Freire, J. R., Fo., Silva, C. B. G., Costa, M. V. da \& Forster, A. C. (2019). Educação Interprofissional nas políticas de reorientação da formação profissional em saúde no Brasil. Saúde em debate, vol. 43, 86-96. https://doi.org/10.1590/0103-11042019s107

Gil, A. C. (2002). Como elaborar projetos de pesquisa. 4, 175. Atlas.

Lei n. 8080, de 19 de setembro de 1990. Dispõe sobre as condições para a promoção, proteção e recuperação da saúde, a organização e o funcionamento dos serviços correspondentes e dá outras providências. Brasília. http://portal.saude.gov.br/portal/arquivos/pdf/lei8080.pdf

Lima, V. V., Ribeiro, E. C. de O., Padilha, R. de Q. \& Mourthé, C. A., Jr. (2018). Desafios na educação de profissionais de Saúde: uma abordagem interdisciplinar e interprofissional. Interface - Comunicação, Saúde, Educação, 22(2), 1549-1562. https://doi.org/10.1590/1807-57622017.0722.

Ministério da Saúde. (2018). Edital $\mathrm{n}^{\circ}$ 10, 23 de julho 2018 seleção para o Programa de Educação pelo Trabalho para a Saúde PETSaúde/Interprofissionalidade - 2018/2019. Diário Oficial União. Brasília, DF.

Ministério da Saúde. (2018). Política Nacional de Educação Permanente em Saúde: o que se produzido para o seu fortalecimento? Brasília. ISBN 978-85-3342649-8

Oliveira, C. M., Batista, N.A., Batista, S. H. S. S. \& Uchôa-Figueiredo, L. R. (2016). A escrita de narrativas e desenvolvimento de práticas colaborativas para o trabalho em equipe. Interface (Botucatu), 20(59), 1005-14. https://doi.org/10.1590/1807-57622015.0660.

Paiva, C. H. A. \& Teixeira, L. A. (2014). Reforma sanitária e a criação do sistema único de saúde: notas sobre contextos e autores. História, Ciências, SaúdeManguinhos, 21(1), 15-36. https://doi.org/10.1590/S0104-59702014000100002

Pereira, A. S., Shitsuka, D. M., Parreira, F. J. \& Shitsuka, R. (2018). Metodologia da Pesquisa Científica. 1, 67. UFSM, NTE.

Silva, J. A. M. da, Peduzzi, M., Orchard, C. \& Leonello, V. M. (2015). Educação interprofissional e prática colaborativa na atenção primária à saúde. Revista da Escola de Enfermagem da Usp, 49(2), 16-24. http://dx.doi.org/10.1590/S0080-623420150000800003. 
Research, Society and Development, v. 10, n. 1, e35510111783, 2021 (CC BY 4.0) | ISSN 2525-3409 | DOI: http://dx.doi.org/10.33448/rsd-v10i1.11783

Silveira, J. L. G. C., Kremer, M. M., Silveira, M. E. U. C., \& Schneider, A. C. T. C (2020). Percepções da integração ensino-serviço-comunidade: contribuições para a formação e o cuidado integral em saúde. Interface (Botucatu), 24, 1-17. https://doi.org/10.1590/Interface.190499 